\title{
The Use of Fuzzy BackPropagation Neural Networks for the Early Diagnosis of Hypoxic Ischemic Encephalopathy in Newborns
}

\author{
Liu Li, ${ }^{1}$ Huo Liqing, ${ }^{2}$ Lu Hongru, ${ }^{1}$ Zhang Feng, ${ }^{2}$ Zheng Chongxun, ${ }^{2}$ Shami Pokhrel, ${ }^{1}$ \\ and Zhang Jie ${ }^{1}$ \\ ${ }^{1}$ Department of Neonatology, Hospital of the Medical College of Xi'an Jiaotong University, Xi'an, Shaanxi 710061, China \\ ${ }^{2}$ Institute of Biomedical Engineering, School of Life Science and Technology, Xi'an Jiaotong University, Xi'an, Shaanxi 710049, China
}

Correspondence should be addressed to Liu Li, nellie918@yahoo.com.cn

Received 1 February 2011; Accepted 6 June 2011

Academic Editor: Vincent C. O. Njar

Copyright (C) 2011 Liu Li et al. This is an open access article distributed under the Creative Commons Attribution License, which permits unrestricted use, distribution, and reproduction in any medium, provided the original work is properly cited.

Objective. To establish an early diagnostic system for hypoxic ischemic encephalopathy (HIE) in newborns based on artificial neural networks and to determine its feasibility. Methods. Based on published research as well as preliminary studies in our laboratory, multiple noninvasive indicators with high sensitivity and specificity were selected for the early diagnosis of HIE and employed in the present study, which incorporates fuzzy logic with artificial neural networks. Results. The analysis of the diagnostic results from the fuzzy neural network experiments with 140 cases of HIE showed a correct recognition rate of $100 \%$ in all training samples and a correct recognition rate of $95 \%$ in all the test samples, indicating a misdiagnosis rate of 5\%. Conclusion. A preliminary model using fuzzy backpropagation neural networks based on a composite index of clinical indicators was established and its accuracy for the early diagnosis of HIE was validated. Therefore, this method provides a convenient tool for the early clinical diagnosis of HIE.

\section{Introduction}

Neonatal hypoxic ischemic encephalopathy (HIE) is a serious illness with a high incidence rate worldwide and can result in death or disability and significantly affect the quality of life. Most current clinical diagnosis methods for HIE are based on medical history and clinical manifestations in combination with imaging techniques. Due to the variability of HIE clinical manifestations and the results of the examinations, these methods are not only time-consuming and costly, but also subject to variations in the experience and skill of the diagnosing physician as well as other subjective factors. These factors make it difficult for HIE to be diagnosed at the early stage. Thus, further research on early diagnosis and intervention of HIE is of great significance for improving the survival, cure rate, and prognosis.

The use of artificial neural networks is a recently developed cross-disciplinary field that integrates neuroscience, information science and computer sciences. It is suitable for the processing of diverse and variable medical data to solve complex issues in the field of medical diagnosis, such as feature extraction. It can eliminate subjective human factors and provide an accurate and objective diagnosis [1-5]. Among many neural network models, the backpropagation (BP) neural network displays a strong learning ability using nonlinear models with a high fault tolerance. It can overcome the deficiencies of traditional medical models and is suitable for pattern recognition and disease diagnosis. In the present study, non-invasive indicators with high levels of early sensitivity and specificity were selected and utilized in fuzzy neural network experiments based on the clinical manifestations of HIE as well as several related studies conducted in China and abroad [6-9].

\section{BP Neural Network Algorithm and Its Improvement}

The first part of the present study focused on improving the optimization of the momentum terms and structure of the BP network, to eliminate the disadvantages of $\mathrm{BP}$ network algorithms such as their liability to fall into a local 
minimum, difficulties in determining the number of hidden layer nodes, slow convergence rate in algorithm learning, poor generalization of the network, and excessive sensitivity to initial values.

2.1. Increasing Momentum Terms. In a standard BP algorithm, weights are adjusted only according to the descending gradient direction of errors at time $t$ without consideration of the gradient direction before time $t$. This setup often leads to oscillations during the learning process and slow convergence. To improve the training speed, a momentum term was added in the weight adjustment formula [1]

$$
\Delta W(t)=\eta \delta O+\alpha \Delta W(t-1),
$$

where $\alpha$ is the momentum coefficient, $0<\alpha<1$.

2.2. Optimization of the Network Structure. The optimization was focused on the number of hidden layers and the number of nodes in each layer of the network.

(1) Number of hidden layers: an increase in the number of hidden layers can form more complex decision-making domains, which can enhance the ability of the network to solve nonlinear problems. An appropriate number of hidden layers can also minimize the system error of the network. Based on the results of a large number of experiments, a three-layered network structure can solve most complex problems.

(2) Number of nodes in the hidden layers: the choice of neuron numbers in the hidden layers is a very complex issue, which is dependent on the experience of the designer and the results from multiple experiments. No optimal analytical expression exists. The number of hidden layer units is directly related to the requirements of the problem and the number of input/output units. If the number is too small, the information obtained through the network will be insufficient to solve the problem. If the number is too large, it will lead to increased training time, longer learning time, a nonoptimal error rate, poor fault tolerance, failure to recognize samples that were not involved in the previous training set, and the possibility of the so-called "transitional agreement" issue. Therefore, the selection of an optimal number of hidden layer units is crucial [10].

The following are the three reference formulas used in this study for the selection of the optimal number of hidden layer units:

$$
\sum_{i=0}^{n} C_{n_{1}}^{i}>k
$$

where $k$ is the sample number, $n_{1}$ is the number of hidden layer units, and $n$ is the number of input units,

$$
n_{1}=\sqrt{n+m}+\alpha,
$$

where $n$ is the number of input units, $n_{1}$ is the number of hidden layer units, $m$ is the number of output units, and $\alpha$ is a constant in the range $[1,10]$,

$$
n_{1}=\log _{2} n,
$$

where $n$ is the number of input units and $n_{1}$ is the number of hidden layer units.

The optimal boundary numbers of the hidden layer units, min and max, were first determined through the incorporated use of formulas (2), (3), and (4). The network training then started from the minimum unit number min, followed by a gradual increase in unit number until the maximum unit number max was validated. For each number of hidden layer units, the network convergence speeds were compared after network convergence was achieved using the same training samples. Finally, the optimal number of hidden layer units was determined based on the training and testing errors of the training results. This approach can effectively reduce the verification time and provide the fastest way to identify the optimal number of hidden layer units.

\section{Structural Design of the Fuzzy BP Neural Network}

The integration of fuzzy systems and neural networks can be classified into five categories based on the form and function of the integration: loose, parallel, series, network learning, and structural equivalence integration.

A series of fuzzy BP networks were employed in the present study, with their frame diagram shown in Figure 1. The entire network $[11,12]$ is composed of two parts, fuzzy processing and a conventional BP network, with the network input the same as a conventional BP network input. Fuzzy processing was performed on the network input through the membership function in the fuzzy processing part, and the processed data were submitted directly to the BP network for further processing. The output data were compared with the expected output and reversely adjusted based on the mean square error to specify the network connection weights.

The structure of a series of fuzzy BP neural networks is shown in Figure 2.

This network consists of five layers. The processing procedure of each layer is as follows.

(1) The first layer is the input layer. The nodes in this layer receive input from the outside and send it to the next layer. The connection weight constant between the first and second layer is 1 .

(2) The second layer is the fuzzification layer. It performs fuzzy processing on the input and calculates the membership function value for each input component.

(3) The third layer is connected to the output of fuzzy processing through the weights. This layer is equivalent to the hidden layer of a three-layered BP network.

(4) The fourth layer is the defuzzification layer. It performs defuzzification processing on the output of the BP network.

(5) The fifth layer is the output layer.

\section{Feature Extraction and Fuzzy Preprocessing}

4.1. Data Source. The medical records of newborns with HIE admitted to our hospital were used as the original raw data. A total of 140 cases were included, with 90 patients and 50 


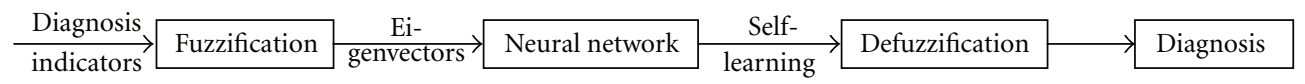

Figure 1: Frame diagram of the fuzzy BP network.

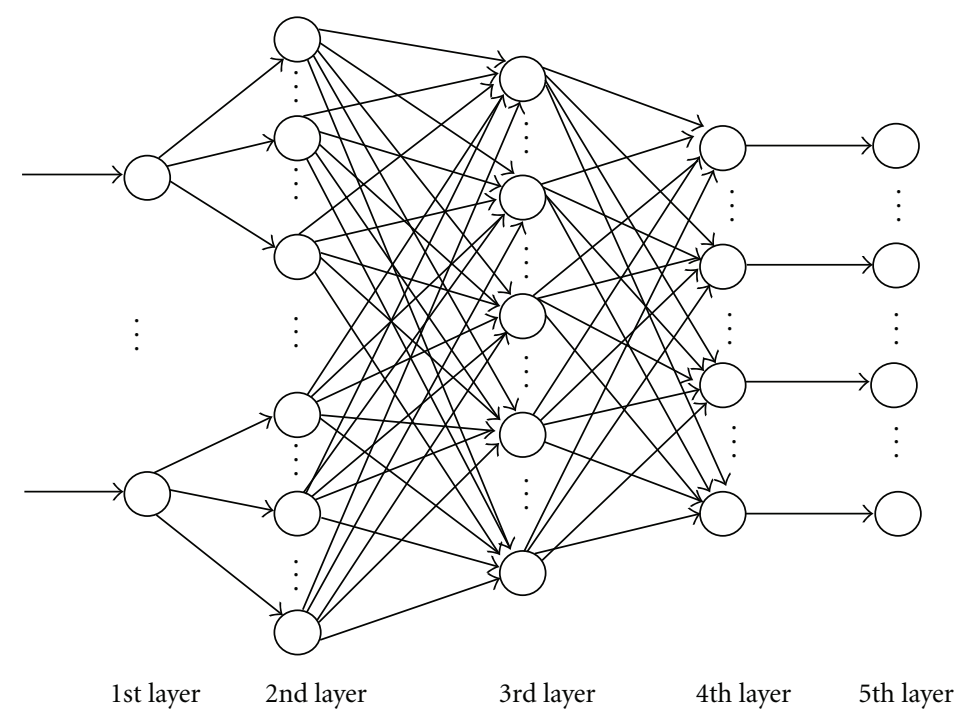

FIGURE 2: The structure of the series of fuzzy BP neural network consisting of five layers.

normal controls. All the HIE cases were diagnosed by group consultation of the experts in the newborn department in our hospital.

4.2. Feature Extraction and Quantification. The present study utilized 10 qualitative and quantitative parameters corresponding to fetal distress, neonatal asphyxia, and HIE clinical manifestations, as well as 15 indicators selected from early diagnostic indicators considered to be important based on several related studies. These indicators, which were used as the input vectors in the first layer, included $\mathrm{Lac} / \mathrm{cr}$ (basal nuclei), NAA/Gr (basal nuclei), DWI (ADC value); (lenticular nuclei), lactic acid/creatinine in urine (the first day), S100B protein $\mathrm{C}$ (the third day), fetal distress (absent, mild, severe), and neonatal asphyxia (absent, mild, severe), as well as eight clinical manifestations that included awareness, muscle tension, embrace reflex, sucking reflex, convulsions, central respiratory failure, pupil changes, and anterior fontanel tension.

To integrate these qualitative and quantitative data, the ten qualitative parameters in the above diagnostic indicators were quantified. The specific quantification for these qualitative indicators is as follows.

(i) Fetal distress: $0=$ absent, $1=$ mild, and $2=$ severe.

(ii) Neonatal asphyxia: $0=$ absent, $1=$ mild, and $2=$ severe.

(iii) Clinical manifestations:

(1) awareness: $0=$ normal, $1=$ slightly excited, $2=$ sleepy and sluggish, and $3=$ coma;

(2) muscle tension: $0=$ normal, $1=$ decreased, and 2 = increased;
(3) moro reflex: $0=$ normal, $1=$ slightly active, $2=$ weak, and 3 = absent;

(4) sucking reflex: $0=$ normal, $1=$ weak, and $2=$ absent;

(5) convulsion: $0=$ absent, $1=$ rare, $2=$ frequent, and 3 = sustained;

(6) central respiratory failure: $0=$ absent, $1=$ mild, and 2 = severe;

(7) pupil changes: $0=$ absent, $1=$ constricted, and 2 = dilated;

(8) anterior Fontanel tension: $0=$ normal, $1=$ slightly tense, and $2=$ tense.

4.3. Selection of Membership Function. Among the commonly used methods for the determination of membership functions, which include the expert evaluation, fuzzy statistical, function approximation, and comparison sorting methods, the main method used in the present study was based on the practical experience of experts, which was gradually improved and optimized through practical tests. Different membership functions were used for different diagnostic indicators. The membership function for each diagnostic indicator is listed below.

(1) Magnetic resonance spectroscopy examination results:

Lac/cr (basal nuclei),

$$
f(x)= \begin{cases}\exp \left(-50(x-a)^{2}\right), & x>a, \\ 1, & x \leq a,\end{cases}
$$

where $a$ is the average of an input indicator. 
TABLE 1: Analysis of the diagnostic results from the fuzzy BP neural network.

\begin{tabular}{lccc}
\hline & $\begin{array}{c}\text { Correct } \\
\text { recognition rate of } \\
\text { training samples }\end{array}$ & $\begin{array}{c}\text { Correct } \\
\text { recognition rate } \\
\text { of test samples }\end{array}$ & $\begin{array}{c}\text { Correct } \\
\text { recognition rate } \\
\text { of all samples }\end{array}$ \\
\hline Normal & $100 \%$ & $100 \%$ & $100 \%$ \\
Mild & $100 \%$ & $100 \%$ & $100 \%$ \\
Moderate & $100 \%$ & $100 \%$ & $100 \%$ \\
Severe & $100 \%$ & $70 \%$ & $95 \%$ \\
\hline
\end{tabular}

NAA/Gr (basal nuclei):

$$
f(x)= \begin{cases}\exp \left(-5(x-a)^{2}\right), & x \leq a, \\ 1, & x>a .\end{cases}
$$

DWI (ADC value) (lenticular nuclei):

$$
f(x)= \begin{cases}\exp \left(-30(\mathrm{x}-\mathrm{a})^{2}\right), & x \leq a \\ 1, & x>a\end{cases}
$$

(2) Urine biochemistry test results:

urine lactic acid/creatinine (the first day),

$$
f(x)= \begin{cases}\exp \left(-0.09(x-a)^{2}\right), & x>a, \\ 1, & x \leq a .\end{cases}
$$

S100B Protein C (the third day),

$$
f(x)= \begin{cases}\exp \left(-0.1(x-a)^{2}\right), & x>a \\ 1, & x \leq a .\end{cases}
$$

For three-level qualitative parameters, normal, mild, and severe were represented by $1,0.5$, and 0 , respectively. For four-level qualitative parameters, normal, mild, moderate, and severe were represented by $1,0.75,0.25$, and 0 , respectively.

\section{Hidden Layer Processing}

In the present study, an improved BP neural network algorithm was used to determine the number of hidden layer nodes. Using (2), (3), and (4), the boundary numbers of the hidden layer nodes were calculated to be 4 and 15 . Network training started at the minimum unit number 4 , until the maximum unit number 15 was reached for verification. The experimental verification showed that the optimal number of hidden layer nodes was 12 .

\section{Design of Defuzzification Layer}

The number of output nodes of the BP network was 4, with an expected output of ( $\left(\begin{array}{llll}1 & 0 & 0 & 0\end{array}\right)$ as normal, $\left(\begin{array}{lll}0 & 0 & 1\end{array}\right.$
$0)$ as mild HIE, (lllll $\left.\begin{array}{llll}0 & 0 & 1 & 0\end{array}\right)$ as moderate HIE, and ( $\begin{array}{ll}0 & 0\end{array}$ 0 1) as severe HIE. Defuzzification was performed based on the corrected maximum membership principles in the defuzzification layer. Since each output node value is either 0 or 1 in the BP network, the node with the maximum value 1 was first identified among the four output nodes, and the output from this node would be the diagnostic result.

\section{Experimental Results and Analysis}

\subsection{Operating Environment}

(1) Software: MATLAB 7.0 programming software (MathWorks), and windows XP operating system.

(2) Hardware: Intel Pentium Dual E2180 $2.00 \mathrm{GHz}$, 3.00 GHz RAM, and $120 \mathrm{~GB}$ HDD PC platform

7.2. Experimental Results and Analysis. A total of 80 cases were randomly chosen from all the samples as the training sets (20 normal controls, 20 cases of mild HIE, 20 cases of moderate HIE, and 20 cases of severe HIE). The remaining 60 cases (30 normal controls, 10 cases of mild HIE, 10 cases of moderate HIE, and 10 cases of severe HIE) were used as test sets.

10 normal controls as well as patients with mild, moderate, and severe HIE underwent test of the 15 diagnostic indicators: Lac/cr (basal nuclei), NAA/Gr (basal nuclei), DWI (ADC value); (lenticular nuclei), urine lactic acid/creatinine (the first day), S100B protein (the third day), fetal distress, neonatal asphyxia, awareness, muscle tension, embrace reflex, sucking reflex, convulsions, central respiratory failure, pupil changes, and anterior fontanel tension.

After treatment, the result of BP network and the final output were displayed. The results of test sample of normal, mild, and moderate were entirely consistent with the clinical diagnosis. The determination of disease level in the moderate and severe cases accounted for most of the diagnostic error. The analysis of the diagnostic results for the 140 samples is shown in Table 1 . The correct recognition rate was $100 \%$ for the training samples, and the correct recognition rate was $95 \%$ for the test samples, indicating a misdiagnosis rate of $5 \%$.

\section{Conclusions}

The fuzzy logic used in a fuzzy neural network was integrated with an artificial neural network in this study. Fuzzy logic can express the logical meanings commonly used by humans in a more natural and direct way. Logical decision-making was performed according to the language rules proposed by experts, which can solve nonlinear questions that cannot be addressed using rigorous modeling methods. The integration of fuzzy logic with a neural network can facilitate selfadaptation through a learning function and automatically acquire an algorithm for the information expressed as fuzzy or precise data. This method can overcome the difficulties in expressing time-varying knowledge and processes, a feature 
of fuzzy logic. The integration of these two components can compensate for the insufficiency of a neural network in fuzzy data processing and the deficiencies of pure fuzzy logic in learning functions $[12,13]$. To construct a fuzzy neural network structure, this system adds a fuzzification layer before the input layer of the neural network and a defuzzification layer after the output layer, which results in the fuzzification of the input information and defuzzification of the output information, respectively. The premises and the confidence level of the conclusions in fuzzy logic, provided by experts in the field, were used as the inputs and expected outputs of the learning samples for the neural network. With the aid of the strong learning and associative memory capabilities of the artificial neural network, the neural network is trained for its learning function to automatically acquire fuzzy rules that are stored in the network in the forms of weights and thresholds. Thus, our fuzzy neural network obtains the capabilities of analyzing fuzzy questions and making diagnoses and achieves an effective combination of fuzzy logic with a neural network.

The introduction of a fuzzy neural network for the early diagnosis of HIE enables the developed system to have better fault tolerance and information processing. During the practical process of determining a specific diagnosis, the developed system processed the fuzzy input information (including fuzzy terms for the description of clinical symptoms such as severe, mild, etc.), and the parallel reasoning of the neural network eliminated the collision problem from the inefficiency of the fuzzy rule matching and rule searching. These abilities enable the system to have a fast reasoning speed and meet the requirements for practical diagnosis. The fuzzy BP neural network used in this study exhibited high accuracy in the early diagnosis of HIE (Table 1), which can provide quantitative indicators for early clinical diagnosis and serve as a convenient diagnostic tool for physicians. In summary, through experiments using the fuzzy neural network, a preliminary data model was established, and its feasibility for early diagnosis was verified. Nevertheless, further research with expanded amount of data should be conducted to increase the accuracy of this method.

\section{Acknowledgment}

This work was supported by the grant of National Natural Science Foundation of China (no. 30772342).

\section{References}

[1] D. Q. Zhu and H. Shi, The Principle and Application of Artificial Neural Network, Science Press, Beijing, China, 2006.

[2] M. J. Er, J. Liao, and J. Lin, "Fuzzy neural networksbased quality prediction system for sintering process," IEEE Transactions on Fuzzy Systems, vol. 8, no. 3, pp. 314-324, 2000.

[3] J. S. Chiu, Y. F. Wang, Y. C. Su, L. H. Wei, J. G. Liao, and Y. C. $\mathrm{Li}$, "Artificial neural network to predict skeletal metastasis in patients with prostate cancer," Journal of Medical Systems, vol. 33, no. 2, pp. 91-100, 2009.

[4] L. Q. Han, The Theory, Design and Application of Artificial Neural Network, Chemical Industry Press, Beijing, China, 2002.
[5] R. F. Harrison and R. L. Kennedy, "Artificial neural network models for prediction of acute coronary syndromes using clinical data from the time of presentation," Annals of Emergency Medicine, vol. 46, no. 5, pp. 431-439, 2005.

[6] L. Liu, H.-Y. Zhou, Z. W. Feng, L. He, and Z. Y. Su, "Urinary S100B protein and lactate/creatinine ratio measurements: a tool for the early identification of neonatal hypoxic-ischemic encephalopathy," Chinese Journal of Pediatrics, vol. 43, no. 8, pp. 564-567, 2005.

[7] L. Liu, H.-Y. Zhou, L. He, J.-W. Song, W.-X. Nie, and Z.Y. Su, "Measuring Urinary S100B protein levels and lactic acid/creatinine ratio for early identification of neonatal hypoxic-ischemic encephalopathy (HIE)," World Journal of Pediatrics, vol. 2, no. 4, pp. 270-275, 2006.

[8] T. Tian, L. Liu, H.-Y. Zhou et al., "The value of urinary S100B protein measurement and urinary lactate/creatinine ratio in predicting the prognosis of full term infants with hypoxicischemic encephalopathy," Chinese Journal of Neonatology, vol. 23, no. 8, pp. 141-144, 2008.

[9] L. Liu, L. He, C. X. Zheng et al., "Application of hydrogen proton magnetic resonance spectrum in hypoxic-ischemic encephalopathy in full-term neonates," Chinese Journal of Pediatrcs, vol. 46, no. 5, pp. 384-385, 2008.

[10] Y. H. Shen, The applied of research neural networks in medical diagnosis. Full-text databaseof China's outstanding, M.S. thesis, 2007.

[11] D. J. Xu, Y. D. Zhou, Y. L. Sang et al., "Research of fuzzy control based on artificial neural network in medicine diagnostic system," Chinese Archives of Traditional Chinese Medicine, vol. 23, no. 5, pp. 781-783, 2005.

[12] X. F. Wan, L. P. Xu, Y. J. Wu et al., "The application of fuzzy neural network to lung cancer diagnosis," Microcomputer Information, vol. 25, no. 2, pp. 295-297, 2009.

[13] H. P. Schwefel, Numerical Optimization of Computer Model, John Wiley \& Sons, Chichester, UK, 1981. 


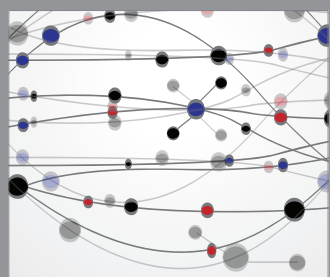

The Scientific World Journal
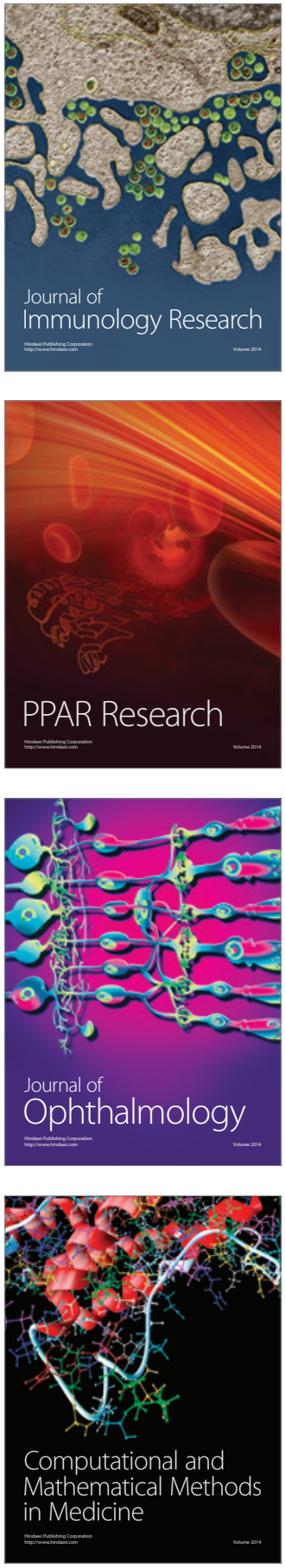

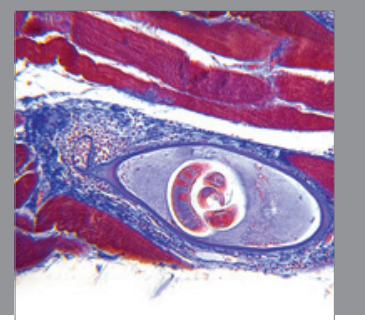

Gastroenterology

Research and Practice
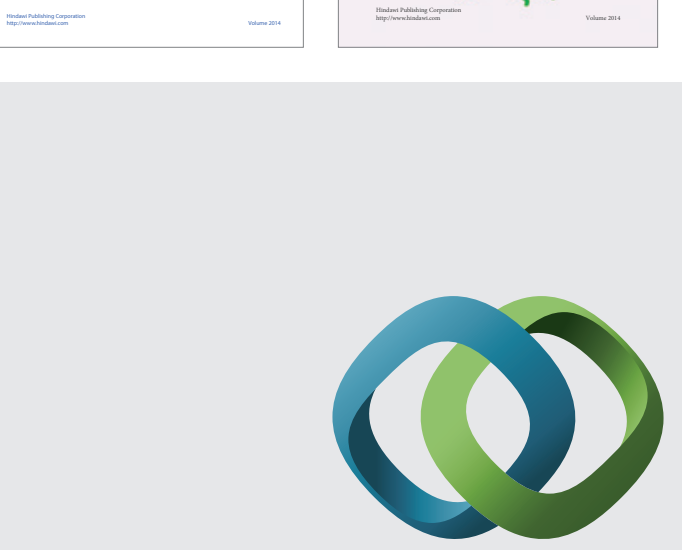

\section{Hindawi}

Submit your manuscripts at

http://www.hindawi.com
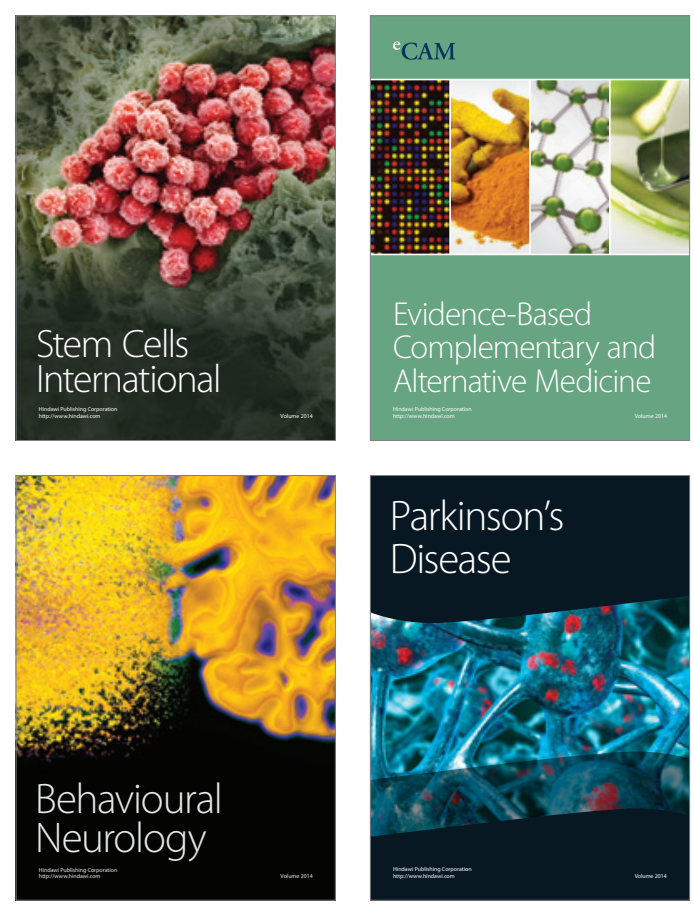

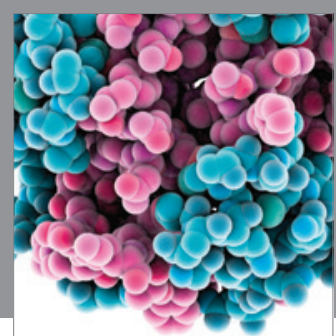

Journal of
Diabetes Research

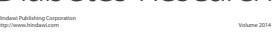

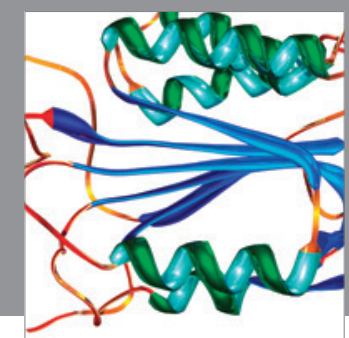

Disease Markers
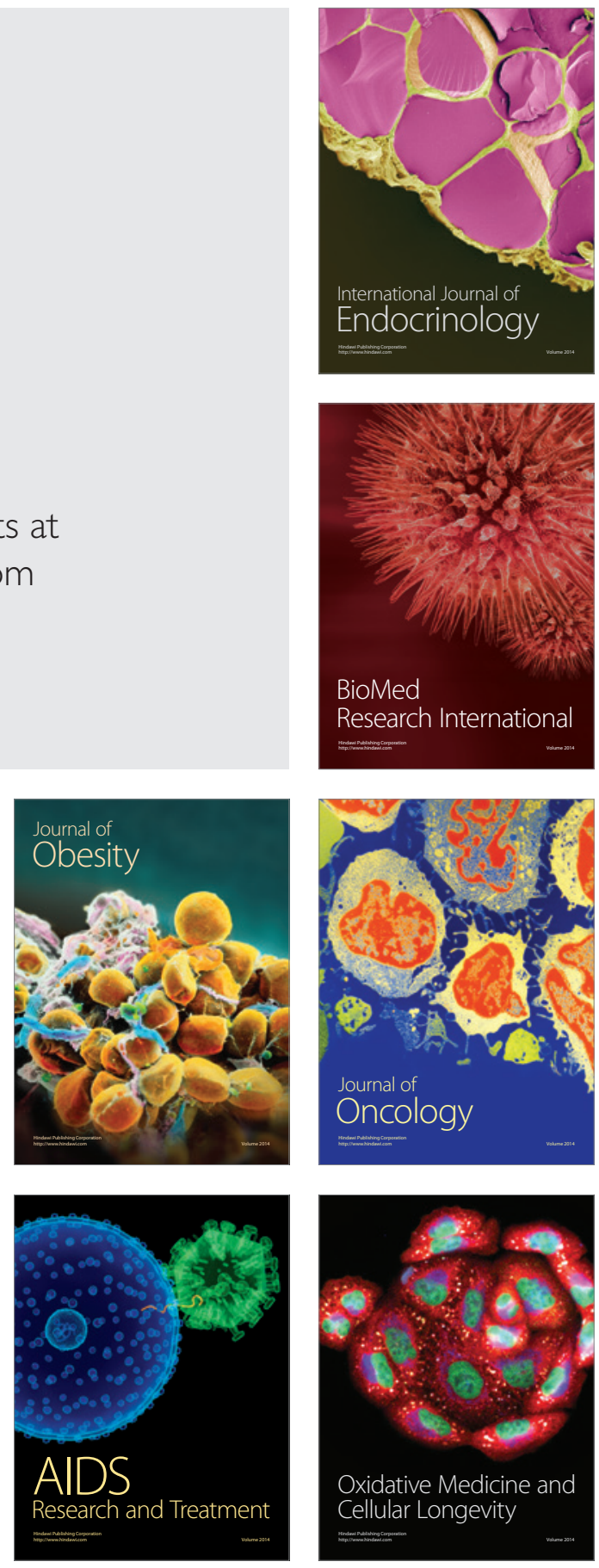